\title{
Development of neotenics induced by a temporary absence of functional reproductives in Kalotermes flavicollis (Isoptera: Kalotermitidae)
}

\author{
JIŘí KINDL and IVAN HRDÝ \\ Institute of Organic Chemistry and Biochemistry, Academy of Science of the Czech Republic, Flemingovo nám. 2 , \\ 16610 Praha 6, Czech Republic; e-mail: kindl@uochb.cas.cz
}

Key words. Inhibition of neotenics, replacement reproductives, temporary absence, Isoptera, Kalotermes

\begin{abstract}
The effect of removing the functional pair of Kalotermes flavicollis from an experimental colony for 12, 24 or $48 \mathrm{~h}$ and the repeated removal for a particular number of hours per day $(2,4,6,12,18,20$, and $22 \mathrm{~h}$ ) was studied. An absence of the functional pair for $12 \mathrm{~h}$ had no affect on the development of new neotenics, whereas 24 -h absence induced the development of new neotenics in 5 out of 12 experimental groups. A 48-h absence induced development of new neotenics in all 12 experimental groups. Pseudergates and nymphs can be orphaned for up to $12 \mathrm{~h}$ a day without being stimulated to differentiate, after which the number of new neotenics increased gradually with the time for which the reproductive pair was absent. This suggests that the inhibitory process is continuous and cumulative. Both sexes showed similar sensitivity to the absence of reproductives. The study also tested, by exchanging pseudergates between groups with functional pairs and orphaned groups, whether pseudergates in experimental groups actively spread inhibitory factors; however, this was not proven. Only pseudergates and nymphs that were in direct contact with the functional pair were inhibited.
\end{abstract}

\section{INTRODUCTION}

The presence of the functional pair in Kalotermes flavicollis colonies prevents the differentiation of neotenics (Grassi \& Sandias, 1893; Lüscher 1974, 1977; Springhetti, 1985). It is thought that this inhibition is realized by means of inhibitory pheromones with the king and queen producing different pheromones with a complementary action (Lüscher, 1952, 1956a,b, 1964). Lüscher (1952) and Springheti (1972) using single and double screens, through which only antennae and odours could pass, showed that the inhibition depends on direct physical contact and not on volatile substances. Pseudergates, larvae and nymphs seem to receive these inhibitory compounds with anal food or through grooming, and distribute them to the other members of the colony in the same way (Lüscher, 1952, 1956a,b). Perhaps the strongest evidence for pheromonal inhibition is that presented by Lüscher (1956a,b), who divided nest in half using wire gauze in which he enclosed reproductives or pseudergates. Inhibition occurred only in the half that included the abdomen of the functional female. Unfortunately, only two females survived this treatment (Lüscher, 1956a). In a similar experiment with pseudergates somewhat higher number survived (Lüscher, 1956b). A significantly smaller number of neotenics developed in the orphaned half with access to the abdominal part of the enclosed pseudergate than in the control. The head of the pseudergate was in the half with the reproductive pair. This finding indicates the active spreading of inhibitory factors by nonreproductive nest-mates.

Removing the functional pair from a colony for only $24 \mathrm{~h}$ can initiate the differentiation of replacement reproductives (Lüscher, 1952). Stuart (1979) mentions that a $48 \mathrm{~h}$ absence of reproductives in Zootermopsis angusticollis (Termopsidae) triggers neotenic development; however, a shorter absence may be sufficient, as he did not try to ascertain the minimum time. Mensa-Bonsu (1976) also showed that in Porotermes adamsoni (Termopsidae) the inhibitory activity of reproductives is ephemeral. Nagin (1972) studied how isolated groups of Neotermes jouteli respond if the reproductive pair is absent for a certain number of hours per day. The level of neotenic production is correlated directly with the time for which the functional reproductives are absent. He suggests that the inhibitory process is continuous and cumulative.

The aim of this study was to understand how pseudergates and nymphs of $K$. flavicollis respond if the reproductive pair is absent from a colony for periods of several hours up to a maximum of two days. The objective of this was to determine for how long inhibitory factors persist after removing the functional pair, and how often members of a colony must be reassured about the presence of the reproductive pair. The aim of exchanging pseudergates between groups with or without functional pair was to investigate if nest-mates actively spread inhibitory factors.

\section{MATERIAL AND METHODS}

Preliminary or definitive groups with replacement reproductives were established three weeks before the start of the experiments. They were checked daily in order to confirm that the groups were under the inhibitory influence of the reproductives. Just before the start of the experiment they were divided into definitive experimental and control groups (see particular scenarios). Breeding neotenic reproductives more than 6 months of age were used as the functioning reproductive pairs. These reproductive pairs originated from the same colony as the termites in the experimental groups.

Termites were kept in Petri dishes (55 $\mathrm{mm}$ in diameter) supplied with pieces of spruce wood $(2$ pieces $30 \times 4 \times 2 \mathrm{~mm}, 2$ pieces $40 \times 4 \times 2 \mathrm{~mm}$ and 1 piece $50 \times 4 \times 2 \mathrm{~mm}$ ). The pieces of wood were glued by their narrow side $5 \mathrm{~mm}$ apart to the bottom of the dish. Humidity was maintained by mean of moist sand in the bottom of the dish and pieces of cellulose wadding, which served also as an additional source of food.

\section{Functional reproductive pair removed once for 12, 24 or} $48 \mathrm{~h}$

Termites: Croatia, Makarska, colony No. 1 and 2, collected 1.vi.2000. 


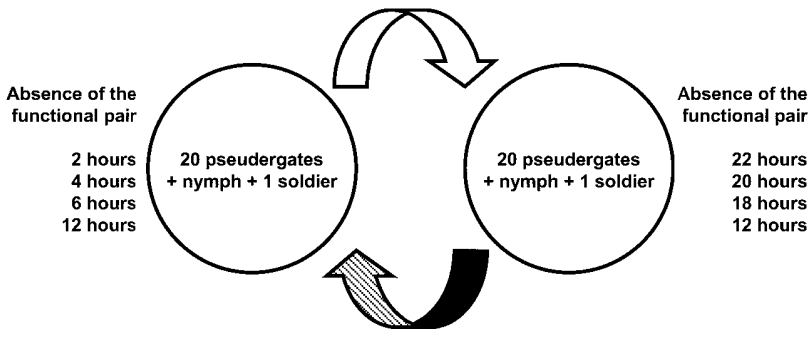

Fig. 1. Diagram of the procedure adopted in the experiments that involved the exchange of the functional reproductives between pairs of experimental groups.

Thirty-six experimental groups were prepared. Each experimental group consisted of a functional reproductive pair, 12 pseudergates and larvae, 8 nymphs and 1 soldier (similar ratio of grown termites as in the original colonies). The functional pair was removed from the experimental groups only once for 12,24 or $48 \mathrm{~h}$ and then returned. Each treatment was repeated 12 times. The day of removal was Day 0 of the experiment. The groups were monitored daily for three weeks and all new replacement reproductives were immediately removed and sexed.

\section{Functional reproductive pair removed temporarily daily}

Termites: Greece, Corfu, Perivoli, colony No. 3, collected 26.v.1999.

This was carried out in two subsequent experiments. In the first the reproductives were removed for 6,12 , or $18 \mathrm{~h}$ daily and in the second for $2,4,20$, or $22 \mathrm{~h}$ daily. The reproductives were repeatedly exchanged between a pair of experimental groups. In each pair the reproductives were left for a fixed period each day in one group and then transferred to the other group where they stayed for the rest of the day. The pattern of the exchanges is given in Fig. 1. This was continued for 36 days and each treatment was repeated 6 times. Each experimental group consisted of 20 pseudergates and nymphs and 1 soldier. The control consisted of six orphaned groups each with the same caste composition. The groups were monitored daily and all new replacement reproductives were immediately removed and sexed.

\section{Exchange of pseudergates between an orphaned group and} one with a functional reproductive pair

Termites: Croatia, Murter, Bethina, colony No. 1 and 2, collected 28.v.2001.

Two experiments were carried out. In the first one, five marked termites were exchanged between an orphaned group and one with a pair of functional reproductives every $12 \mathrm{~h}(10$ termites per $24 \mathrm{~h}$ ) for 36 days. Both orphaned groups and groups with reproductives consisted of 20 pseudergates and nymphs, and 1 soldier, and 5 marked pseudergates. In order to maximize the probability of contact between particular termites and the reproductives a different arrangement was adapted in the second experiment. Ten marked pseudergates were exchanged between groups of orphaned termites ( 20 pseudergates and nymphs, and 1 soldier) and a group with a functional pair of neotenics and 1 soldier every $12 \mathrm{~h}$ (20 termites per $24 \mathrm{~h}, 10$ red and $10 \mathrm{blue}$ ) for 36 days. The exchanged (marked) termites were considered as one category in the statistical analysis. Both studies lasted 36 days and were each repeated seven times. The exchanged termites were topically marked with Herlitz fix. The marked termites seemed to integrate into the experimental groups. They were always distributed among the experimental termites (their original nest mates) and some social interactions were observed. However, in order to avoid disturbance of the experimental
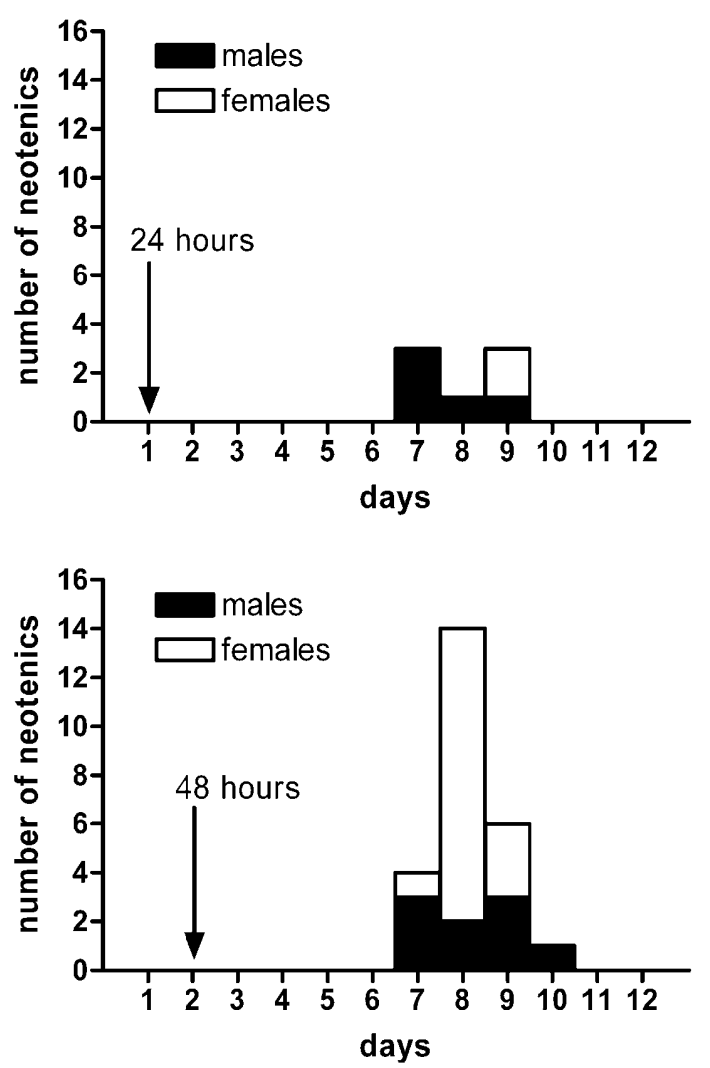

Fig. 2. Effect of the absence of the functional reproductive pair for 24 or $48 \mathrm{~h}$ on the development of new neotenics. Twelve replications. The reproductive pair was removed on day 0 . Arrows show when the functional pair was returned to the colony.

groups they were not subject to detailed observation. Groups were monitored daily and all new neotenics were immediately removed and sexed. Seven orphaned groups of the same caste composition as the experimental ones served as the control.

\section{Statistics}

Data were analysed using ANOVA and Tukey's Multiple Comparison Test. All analyses employed Graph Pad Prism version 3.02 for Windows, Graph Pad Software, San Diego California USA, www.graphpad.com.

\section{RESULTS}

\section{Functional reproductive pair removed once for 12,24 or $48 \mathrm{~h}$}

The number and sex of newly developed neotenics is presented in Fig. 2. A $12 \mathrm{~h}$ absence of the functional pair was not long enough to trigger the development of new neotenics. A 24-h absence induced the development of new neotenics in 5 of the 12 experimental groups. In total 5 male and 2 female neotenics developed. Neotenics developed between the 7th and 9th day of the experiment. A $48 \mathrm{~h}$ absence induced development of new neotenics in all 12 experimental groups. Nine male and 16 female neotenics developed. They developed between the 7 th and 10th day of the experiment.

\section{Functional reproductive pair removed temporarily daily}

The number and sex of the newly developed neotenics are presented in Fig. 3 and 4. Two to $12 \mathrm{~h}$ of absence daily had no or only a minimal impact on the development of neotenics (in both the 6 and $12 \mathrm{~h}$ treatment only 1 neotenic developed). New 

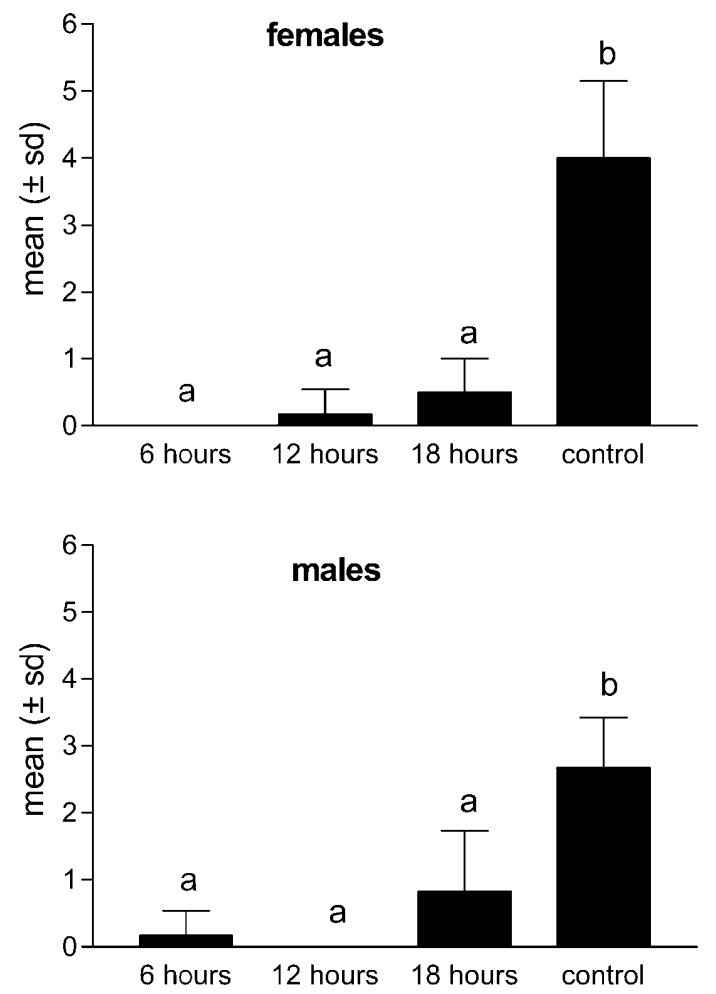

Fig. 3. Effect of the absence of the functional pair for 6,12 or $18 \mathrm{~h}$ per day on the development of new neotenics. Six replications. Females: ANOVA, $\mathrm{F}_{(3,20)}=41.94 ; \mathrm{P}<0.0001$, males: ANOVA, $\mathrm{F}_{(3,20)}=19.88 ; \mathrm{P}<0.0001$. Columns with the same letters are not significantly different. Tukey's Multiple Comparison Test.

neotenics developed in small numbers after $18 \mathrm{~h}$ of absence daily. Twenty hours of absence daily inhibited slightly the development of female neotenics. They appeared in significantly smaller numbers compared to the control. The number of new male neotenics after $20 \mathrm{~h}$ and number of both sexes after 22 $\mathrm{h}$ of absence daily of reproductives were not different from the number of neotenics in fully orphaned control groups.

Exchange of pseudergates between a group with a functional reproductive pair and an orphaned group

The number and sex of the newly developed neotenics are presented in Figs 5 and 6.

Exchange of 5 marked pseudergates every $12 \mathrm{~h}$ between an experimental group with a functional pair and an orphaned experimental group

There was no significant difference in the number of newly developed neotenics in the control orphaned groups and groups of termites that were in contact for $12 \mathrm{~h}$ with 5 termites that had previously spent $12 \mathrm{~h}$ in an experimental group with a functional reproductive pair. None of 10 exchanged termites developed into neotenics in any of the 7 replicates.

Exchange of 10 marked pseudergates every $12 \mathrm{~h}$ between the functional pair and an orphaned experimental group

Only those pseudergates and nymphs that were in direct contact with the functional pair (exchanged termites were considered as one category) were inhibited. There was no significant difference in the number of the newly developed neotenics between the control (orphaned group) and groups of termites
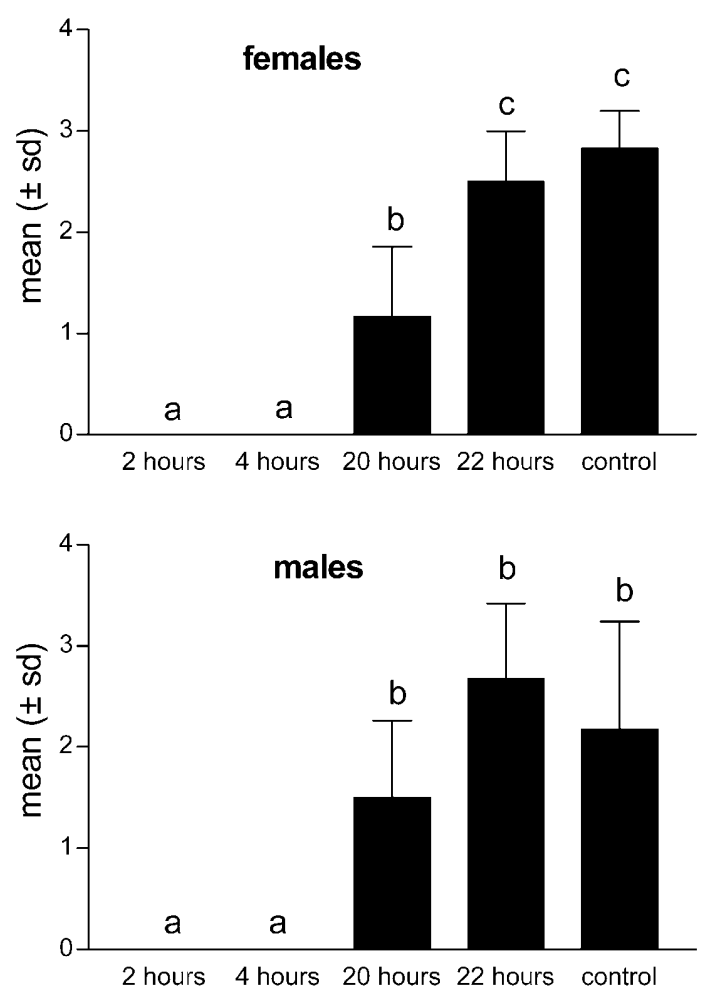

Fig. 4. Effect of the absence of the functional pair for 2, 4, 20 or $22 \mathrm{~h}$ per day on the development of new neotenics. Six replications. Females: ANOVA, $\mathrm{F}_{(4,25)}=52.18 ; \mathrm{P}<0.0001$, males: ANOVA, $\mathrm{F}_{(4,25)}=16.55 ; \mathrm{P}<0.0001$. Columns with the same letters are not significantly different. Tukey's Multiple Comparison Test.

that were in contact for $12 \mathrm{~h}$ with exchanged termites that had previously been in contact with the functional pair for $12 \mathrm{~h}$ ).

\section{DISCUSSION}

While a $12 \mathrm{~h}$ absence of the functional pair did not influence their inhibitory action, a $24 \mathrm{~h}$ absence induced the development of 7 new neotenics in 5 of the 12 experimental groups and a 48 $\mathrm{h}$ absence initiated development of neotenics in all the experimental groups. Lüscher (1952) recorded 7 male neotenics in 4 groups out of 12 after a $24 \mathrm{~h}$ absence of the functional pair. In contrast, female neotenics ( 2 individuals) also developed in the experiment described here. In both, the 24 and $48 \mathrm{~h}$ absence experiments, new neotenics developed on the 7th to 10th day of the experiment. There was no noticeable shift in the time of development of new neotenics with increase in period of absence. Male pseudergates seem to be more responsive to the absence of the reproductive pair than female pseudergates.

Hence, a 24-h cycle was chosen for measuring the response of Kalotermes flavicollis groups to the repeated absences of reproductives. The use of a 24-h cycle also allowed the results to be compared with these of Nagin (1972). Natural nests of $K$. flavicollis lack a royal cell with a special microclimate and social regime, and the pair can theoretically move freely throughout the colony. Nonetheless, Springhetti (1980), showed that reproductive pairs of $K$. flavicollis tend to inhabit a definite area of an artificial nest as well as they tend to stay near the "nursery area" under natural conditions. In spite of this it is hoped, that the transfer of reproductives is not so stressful to influence the release of the presumed inhibitory factor. Pseudergates and nymphs could be orphaned for up to $12 \mathrm{~h}$ per day without being 

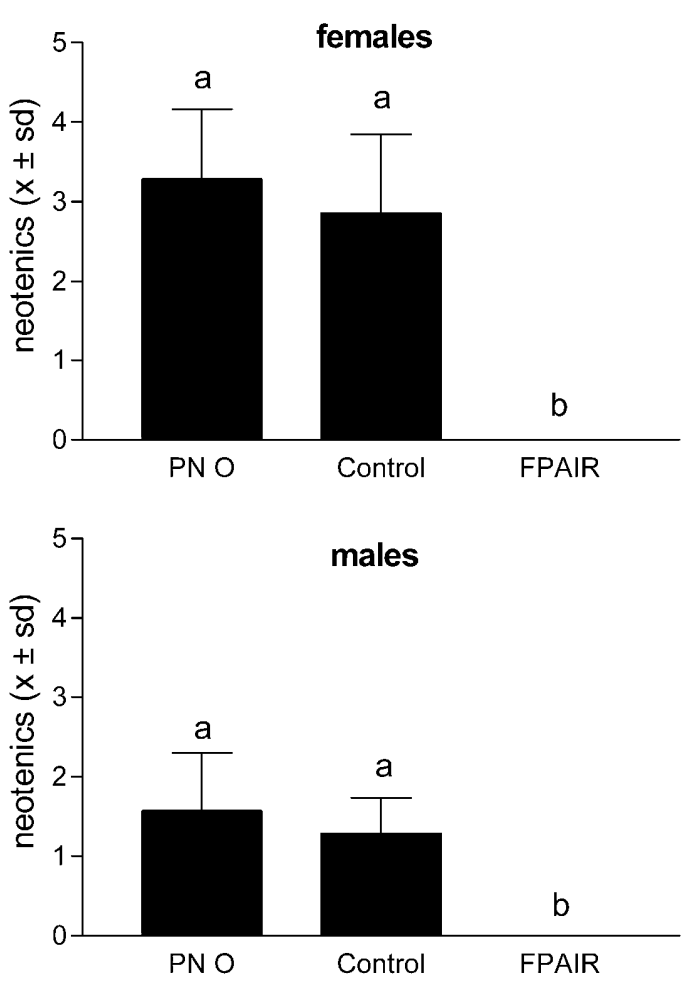

Fig. 5. Number of newly developed neotenics in experimental groups after exchanging 5 marked pseudergates every $12 \mathrm{~h}$ for 36 days between an experimental group with (FPAIR) and without (PN O) a functional pair. Seven replications. Inhibition of neotenics was recorded only in the groups with a functional pair. Females: ANOVA, $\mathrm{F}_{(2,18)}=32.72 ; \mathrm{P}<0.0001$, males: ANOVA, $\mathrm{F}_{(2,18)}=17.17 ; \mathrm{P}<0.0001$. Columns with the same letters are not significantly different. Tukey's Multiple Comparison Test.

stimulated to differentiate into neotenics. Then the number of new neotenics increased gradually with the increase in the number of hours the reproductives were absent. This fact suggests that the inhibitory process is continuous and cumulative. These findings concur with those of Nagin (1972). Differentiation into neotenics depends on where an individual is in the moulting cycle. The susceptibility is highest shortly after a moult (Lüscher, 1952). There may also be individual variation in the threshold for the inhibitory factor. The duration of absence that was successfully compensated for by a subsequent period of contact with reproductives (using a $24 \mathrm{~h}$ cycle) is at least $12 \mathrm{~h}$ then neotenics start to develop. In the case of female neotenics, 6 or $4 \mathrm{~h}$ of daily presence of the functional reproductives significantly inhibits their development compared to the controls. This means that within these periods a number of events, or interactions between reproductives and pseudergates must occur, which affect the initiation of neotenic development. These events might correlate with grooming or proctodeal trophallaxis. Both sexes showed a similar sensitivity to the absence of the reproductives, contrary to the results obtained by Nagin (1972) using Neotermes jouteli, where male pseudergates were much more sensitive to a shorter absence of the reproductive pair than the female pseudergates. The response of females of Neotermes jouteli was similar to that of Kalotermes flavicollis. This difference may be ascribed to species specificity. Other species-specific differences in the neotenics in the lower termites are mentioned by Nagin (1972), Stuart (1979) and Myles (1999).
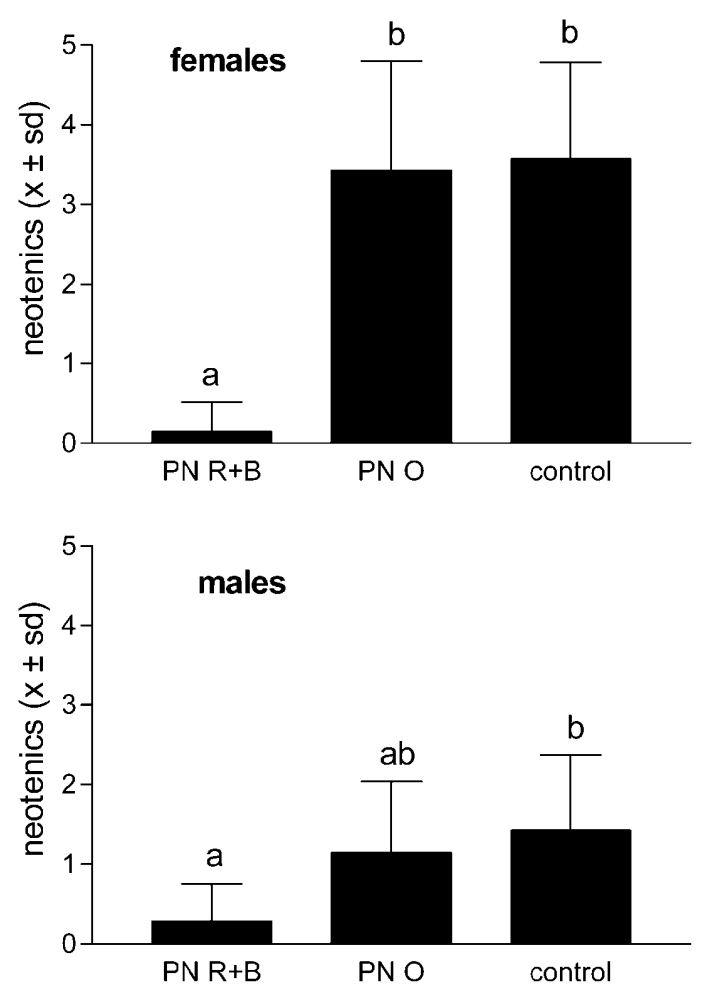

Fig. 6. The number of neotenics that developed in experimental groups after exchanging 10 marked pseudergates every $12 \mathrm{~h}$ for 36 days between a group with a functional pair of reproductives and an orphaned group. Seven replications. PN $\mathrm{R}+\mathrm{B}$ - exchanged termites (red and blue marks, considered as one category), $\mathrm{PN} \mathrm{O}$ - orphaned experimental group, control (totally orphaned group). Females: ANOVA, $\mathrm{F}_{(2,18)}=32.72 ; \mathrm{P}<$ 0.0001, males: ANOVA, $\mathrm{F}_{(2,18)}=17.17$; $\mathrm{P}<0.0001$. Columns with the same letters are not significantly different. Tukey's Multiple Comparison Test.

Termites that were in contact with the functional pair for $12 \mathrm{~h}$ a day were inhibited but unable to transmit the inhibitory factor to other termites in orphaned group over a period of $12 \mathrm{~h}$. By enclosing a pseudergate in a screen between orphaned pseudergates and pseudergates with a functional pair, Lüscher (1956b) showed that some inhibitory activity was distributed via this "enclosed" pseudergate. If this occurs via proctodeal trophallaxis or grooming, then it is somewhat difficult to imagine how this could have operated as the recipient in proctodeal trophallaxis always initiates the act (Maistrello \& Sbrenna, 1998, 1999, and pers. observ., unpubl.) and the "enclosed" pseudergate could not move.

It seems that in small experimental colonies the inhibition of the development of neotenics is achieved mainly through direct contact with the reproductive pair.

ACKNOWLEDGEMENTS. I wish to thank M. Lenz and three anonymous referees for valuable comments on the manuscript. This work was supported by grant No S4055104 GA of Academy of Sciences of the Czech Republic and research project Z4-055-905 (Academy of Sciences of the Czech Republic).

\section{REFERENCES}

Grassi B. \& Sandias A. 1893: Constituzione e sviluppo della societá die Termitidi. Atti Accad. Gioenia, Catania 6/7: $1-150$. 
LÜSCHER M. 1952: Die Produktion und Elimination von Ersatzgeschlechtstieren bei der Termite Kalotermes flavicollis Fabr. Z. Vergl. Physiol. 34: 123-141.

LÜSCHER M. 1956a: Die Entstehung von Ersatzgeschlechtstieren bei der Termite Kalotermes flavicollis Fabr. Insectes Soc. 3 119-128.

LÜSCHER M. 1956b: Hemmende und fördernde Faktoren bei der Entstehung von Ersatzgeschlechtstieren bei der Termite Kalotermes flavicollis Fabr. Rev. Suisse Zool. 63: 261-267.

LÜSCHER M. 1974: Kasten und Kastendifferenzierung bei niederen Termiten. In Schmidt G.H. (ed.): Socialpolymorphismus bei Insekten. Wissenschaftl. Verlaganst., Stuttgart, pp. 694-739.

LÜSCHER M. 1977: Queen dominance in termites. Proc. VIIIth Congr. I.U.S.S.I. Wageningen, pp. 238-242.

Maistrello L. \& Sbrenna G. 1998: Behavioral profiles in laboratory colonies of Kalotermes flavicollis (Isoptera: Kalotermitidae) with different social environments. Sociobiology 31: 91-104.

Maistrello L. \& Sbrenna G. 1999: Behavioural differences between male and female replacement reproductives in Kalotermes flavicollis (Isoptera: Kalotermitidae). Insectes Soc. 46: 186-191.
Mensa-Bonsu A. 1976: The production and elimination of supplementary reproductives in Porotermes adamsoni (Froggat) (Isoptera, Hodotermitidae) Insectes Soc. 23: 133-153.

Myles T.G. 1999: Review of secondary reproductives in termites (Insecta: Isoptera) with comments on its role in termite ecology and social evolution. Sociobiology 33: 1-91.

NAGIN R. 1972: Caste determination in Neotermes jouteli (Banks). Insectes Soc. 19: 39-61.

SpRINGHETti A. 1972: I reali nella differenziazione delle caste di Kalotermes flavicollis (Fabr.) (Isoptera). Boll. Zool. 39: 83-87.

Springhetti A. 1980: A royal arena in the nest of Kalotermes flavicollis (Fabr.). Monitore Zool. Ital. (N.S.). 14: 53-61

SpRINGHetti A. 1985: The function of the royal pair in the society of Kalotermes flavicollis (Fabr.) (Isoptera: Kalotermitidae). In Watson J.A.L., Okot-Kotber B.M. \& Noirot C. (eds): Caste Differentiation in Social Insects. Pergamon Press, Oxford, pp. 165-175.

Stuart A.M. 1979: The determination and regulation of the neotenic reproductive caste in the lower termites (Isoptera): with special reference to the genus Zootermopsis (Hagen). Sociobiology 4: 223-237.

Received March 8, 2004; revised August 24, 2004; accepted September 27, 2005 